\title{
Maßstäbe einer Verfassung auch für Frauen - eine andere Freiheit, Gleichheit, Würde
}

Wer hätte vor fast genau einem Jahr, als wir uns am gleichen Ort an die Rolle und die Erfahrungen von Frauen in der Französischen Revolution erinnerten, ${ }^{1}$ geahnt, daß wir so bald ähnliche Erfahrungen machen bzw. so unmittelbar Zeuginnen einer gleichen Geschichte werden würden: Wenn es darum geht, die Tyrannen zu stürzen - in der revolutionären Bewegung, die zum > Volk < wird - sind die Frauen dabei und zwar in der vordersten Reihe und in großer Zahl. Doch beim Einsammeln der Früchte der Revolution, bei der politischen Gestaltung und Entscheidung über die so veränderten Verhältnisse, führen Männer das Wort, machen ihr groBes Geschäft, heften sich die Verdienste anderer - in diesem Fall der BürgerInnenbewegung in der DDR - an ihre viel zu breiten, patriarchalen Schultern.

Wieder einmal haben uns Männer vorgeführt, wie sie beanspruchen, allein die Geschichte zu machen. Die Lasten und Kosten auch dieser Umwälzung aber tragen die Frauen: Die Frauen in der DDR, indem sie als erste ihre Arbeitsplätze, ihr Recht auf Erwerb, ihr Recht auf Bildung und Ausbildung trotz und neben ihren Familienpflichten, aber auch die unzureichenden Kompensationen für die doppelte Aufgabe, insbesondere die Vorkehrungen für die Kinderbetreuung verlieren. Und die Frauen in der (alten) BRD bleiben wie eh und je aufgerufen, ihre angeblichen Sonderinteressen zurückzustellen, z.B. unsere dezidiert ausgearbeitete Kritik an den unsozialen Folgen einer »sozialen Marktwirtschaft — - »kostenneutral « - dem höheren Ziel der Einheit des , Vaterlandes< zu opfern. Doch »wir Frauen haben kein Vaterland « - so schon eine politische Schrift aus der Frauenbewegung am Beginn des Jahrhunderts. ${ }^{2}$ An seinem Ende fügen wir hinzu: Wir wollen kein Vaterland! Keinen Nationalismus, kein starkes, mächtiges Deutschland, dessen GroBmannssucht seinen Nachbarn Angst macht. Davon haben wir genug! Und wir sind nicht »das Volk«, das zu diesem Ende einer weltbewegenden und dennoch friedlichen Revolution in Deutschland die Begleitmusik spielt. Aber wer sind wir, was wollen wir, in welchen Begriffen, mit welchen Mitteln können wir unseren Forderungen, unserem >Traum von Menschenglück` Ausdruck verleihen?

Nachdem der erste Jubel über neue Freiheiten und Freizügigkeiten verraucht war, schienen wir anfangs wie gelähmt, sprachlos geworden. Die erneute Erfahrung des Ausschlusses von Frauen, der Ausschaltung der Frauen in der DDR, die 
die revolutionäre Bewegung angeführt, so viel Mut bewiesen und uns Mut gemacht haben, sowie die Fast-Nichtbeteiligung von Frauen in den Ausschüssen und Gremien, in denen das Verfahren der Vereinigung, richtiger wohl: der Vereinnahmung ausgehandelt wurde. Doch dann packte uns Wut, Empörung - und auch um der Resignation zu entgehen, haben wir beschlossen, etwas zu tun, auf unsere Weise und mit Eurer/lhrer aller Hilfe. Denn Nichtstun heiBt Geschehenlassen und doch Mitverantworten!

Der Einigungsvertrag mit seinen 45 Artikeln, Zusatzprotokollen und Anlagen, einem Paragraphenberg von Überleitungs- und Anpassungsbestimmungen an die Rechtsordnung der BRD, ist ein Gesellenstück juristischer Fachkompetenz und Perfektion - ebenso bürokratisch wie bürgerfern. Im Eilverfahren - unter der erpresserischen Androhung, die Einheit zu gefährden - durch die parlamentarischen Gremien gejagt, fehlt ihm dennoch die demokratische Legitimation.

Die Notwendigkeit, unsere gemeinsame und seit 40 Jahren getrennte Geschichte nicht nur im Munde zu führen, sondern aus der Trauerarbeit um zugefügtes Leid politische Konsequenzen zu ziehen, die einzigartige Chance, unter den Bewohnern der beiden Teile Deutschlands eine selbstbestimmte und solidarische Vereinbarung über die wichtigsten Regeln des Zusammenlebens, den Ausgleich krassester Ungleichheiten zu treffen, wurde da vertan. Ja, die Vorschrift des noch geltenden Verfassungsrechts, des Art. 146 GG im Falle der Einheit Deutschlands eine Verfassung »für das gesamte Deutsche Volk« zu diskutieren, »in freier Entscheidung « zu beschließen und in Kraft zu setzen, ist mit dem Federstrich eines einfachen Gesetzes von der politischen Tagesordnung gewischt worden. Verfassungsfragen sind gemäB Art. 5 des Einigungs-Vertrages nur noch als Verfassungsänderungen oder -ergänzungen vorgesehen. An die Stelle des konstitutiven Verfassungsaktes tritt ein AnschluBvertrag, bei dem der Stärkere die Bedingungen diktiert hat, im wahrsten Sinne des Wortes das Recht des Stärkeren gilt, anstatt das »Wohl der Schwachen zum Maßstab geltenden Rechts« zu machen (so in der Vorrede zur Schweizer Verfassung).

Dabei wäre die so männlich dominierte Vereinigung zuguterletzt beinahe noch an einer Frauenfrage gescheitert, die schnell zur "Nebensächlichkeit« erklärt wurde: Die unterschiedliche Rechtslage zur Strafbarkeit des Schwangerschaftsabbruchs, die offenbar einen nicht nur symbolisch, sondern auch existentiell >wunden Punkt` im gegenwärtigen Verhältnis der Geschlechter bezeichnet. Was muß dies für ein Punkt sein, in dem das ungelöst Problematische im Geschlechterverhältnis nun seit 100 Jahren immer wieder so grundsätzlich aufbricht? - Ich komme darauf zurück.

Ernstzunehmen ist der Einwand, ob die Einmischung in eine so abstrakt und so fern von den Interessen der Frauen geführte Debatte um die Verfassung des künftigen Deutschland nicht ein müßiges, sinnloses Unterfangen ist, bei dem wir »unsere Energien verpulvern ${ }^{3}$ und in Anbetracht der wieder einmal bestätigten Machtverhältnisse zwischen Frauen und Männern ohnehin nichts ausrichten können. Richtig ist, daB der juristische Weg nun umständlicher, aber auch nicht unpassierbarer geworden ist: Er setzt voraus, daß eine Zweidrittelmehrheit unseres Parlaments eine verfassungsgebende Versammlung einberuft und damit den Weg zur Neuschöpfung der Verfassung freigibt. Eine sehr unwahrscheinliche Varian- 
te? Nun, sind wir nicht zusammen mit den anderen Initiatoren für eine Verfassung mehr als die Hälfte des Wahlvolks? SchlieBlich sieht selbst der Einigungsvertrag (Art. 4 Abs. 6) vor, daB das »Grundgesetz ... nach Vollendung der Einheit und Freiheit Deutschlands ... seine Gültigkeit an dem Tage (verliert), an dem eine Verfassung in Kraft tritt, die von dem deutschen Volke in freier Entscheidung beschlossen worden ist.k

In der mittlerweile von verschiedenen Seiten geforderten Verfassungsdebatte aber geht es nicht um Abstraktionen oder feierliche Grundsätze. Die Verfassung eines Staates ist keineswegs nur eine Angelegenheit der juristischen Zunft und anderer Experten. Sie ist nicht nur als ein Organisationsstatut für die Organe und das Handeln des Staates zu verstehen. Als einklagbarer Wertekanon hat das Bundesverfassungsgericht in den vergangenen 40 Jahren nicht von ungefähr die Schiedsrichterrolle in politischen Fragen übernommen, insbesondere auch im Konflikt zwischen Männern und Frauen, und wie alle Juristinnen wissen, keineswegs immer zuungunsten der Frauen. Doch darüberhinaus verstehe ich die Initiative zu einer Diskussion über unsere zukünftige Verfassung dieses neuen deutschen Gemeinwesens als Zwischenstation zu einer europäischen Gemeinschaft gerade in der Zeit des Umbruchs, der Unsicherheiten, aber auch der neuen Möglichkeiten als notwendigen Verständigungsproze $B$ nicht nur zwischen den Menschen in West und Ost, sondern auch zwischen Männern und Frauen.

Denn es geht, auch noch nach dem 3. Oktober 1990, um die Neuorganisation unserer politischen Verhältnisse, die Regeln für das Zusammenwachsen zweier unterschiedlicher Gesellschaftssysteme, um die Diskussion der Maßstäbe für Recht und soziale Gerechtigkeit, um eine gerechtere Verteilung der Arbeit und der Güter und um die Heilung und Schonung unserer Umwelt und unserer sog. natürlichen Lebensgrundlagen, schließlich um unumstößliche Vorkehrungen für den Frieden mit unseren Nachbarn und in der Welt - aber auch um so Alltägliches wie die endliche Verhinderung von Gewalt gegen Frauen und Kinder und um die Lösung vieler sozialer Probleme nicht wiederum auf Kosten der Frauen. Dabei haben wir ganz ausdrücklich nicht nur die Durchsetzung und Verbesserung von Frauenrechten im Sinn - auch wenn ein Erfolg in dieser Hinsicht schon viel, mehr als die Hälfte wäre - vielmehr zielt unser Unternehmen auf's Ganze, auf alle Bereiche der Gesellschaft und ihrer Verfassung, auf das zur Geltungbringen weiblicher Erfahrungen und Wertmaßstäbe, ja, auf die Einsicht, daß Frauenfragen immer auch gleichzeitig Männerfragen sind, weil Geschlechterpolitik in der Geschichte des Kapitalismus wie des jetzt unrealen Sozialismus das Fundament gesellschaftlicher Ungleichheit war und ist.

Aus diesem Grund setzen wir uns dafür ein, auf der Basis unserer guten und schlechten Erfahrungen mit dem Grundgesetz, ermutigt und belehrt durch die BürgerInnenbewegung der DDR und den Verfassungsentwurf des $>$ Runden Tisches` eine neue Verfassung zu entwerfen und damit einen Gesellschaftsvertrag zu schließen zwischen Frauen und Männern.

Wenn ich Frauenfragen selbstverständlich als Gesellschaftsfragen begreife, von weiblichen Erfahrungen und Bewertungen spreche, so stützen sich mein Mut und die Überlegungen hierzu auf das Politikverständnis der Frauenbewegung. Diese seit dem Beginn der 70er Jahre neue Emanzipationsbewegung der Frauen, 
die weltweit ihren Protest gegen geschlechtshierarchische Ungleichheiten trotz formaler Rechtsgarantien anmeldete, das Selbstbestimmungsrecht der Frauen einklagte und die offene wie die im Privaten verborgene Gewalt gegen Frauen als Politikum anprangerte, hat im Verhältmis der Geschlechter, in den privaten wie gesellschaftlichen Beziehungen mehr BewuBtseins-, wenn auch noch nicht Verhaltensänderungen bewirkt, als ihre Gegner wahrhaben wollen. Und obwohl sie als autonome Bewegung gerade in der Bundesrepublik in bewuBter Abkehr von den etablierten Politikformen zunächst nicht in Vereinen oder Parteien organisiert ist, ihre Projekte chronisch unter Geldmangel leiden, ihre Unorganisiertheit bei vielen Anlässen Schwäche und Stärke zugleich ist, haben zunehmend doch frauenbewuBte Frauen den Weg in und durch die Institutionen angetreten, um - mit Frauen und Freundinnen im Rücken - Männem die Machtfrage zu stellen. Auf diese Weise erfaBt die Frauenbewegung weit mehr als diejenigen, die sich selbst als Feministinnen bezeichnen. Zugleich aber läßt der ProzeB der Verbreiterung und Ausdifferenzierung die unterschiedlichen Auffassungen und Orientierungen auch zwischen Frauen sehr viel deutlicher hervortreten. In einem oft schmerzlichen und mühsamen ProzeB versuchen wir mittlerweile unsere Differenzen zu erkennen, sie anzuerkennen und deshalb besser mit ihnen umzugehen. Doch gerade bei der Festlegung auf eine bestimmte Politik in der Form des Rechts brechen die Unterschiede und Mißverständnisse wieder auf, will es uns nicht ohne weiteres gelingen, gemeinsam stark zu sein. Um welche Positionen es hierbei geht, möchte ich im folgenden am Beispiel der drei grundlegenden Begriffe und Rechtskategorien "Freiheit, Gleichheit und Würde « skizzieren, in der Absicht und Hoffnung, hiermit zur Vorverständigung über unseren weiteren Rechtekatalog und seine Diskussion am heutigen Tag beizutragen. ${ }^{4}$

\section{Freiheit}

Freiheit hat in unserer Rechtstradition einen hehren Klang, erst recht für eine Befreiungsbewegung wie die der Frauen. Und doch ist die Frage, wieviel Freiheit sich jede und jeder nehmen kann, das Grundproblem aller Rechtstheorie. Denn eine unbeschränkte Handlungsfreiheit kann es in einer Gesellschaft der vielen, die Rücksichtnahme und Gegenseitigkeit erfordert, nicht geben. Das Problem aber besteht darin, daB die Bedingungen der Freiheit für die Einzelnen unterschiedlich sind. Das war der Inhalt der Marx'schen Kritik an der doppelten und "zwieschlächtigen « Freiheit des Lohnarbeiters. Dies ist der Grund, warum Freiheit nur in Verbindung oder in einem Spannungsverhältnis mit Gleichheit zur Leitnorm der Menschenrechte avancierte. "Alle Menschen sind frei und gleich an Würde und Rechten«, heißt die Formulierung der UNO-Deklaration. Nach herrschender Meinung hat die Freiheit im Wertsystem des Grundgesetzes jedoch einen Vorrang gegenüber der immer noch des Sozialismus verdächtigen Gleichheit, wenn auch betont wird, daß die Freiheit des Art. 2 GG nur als »sozialgebundene Entfaltungsfreiheit« Sinn macht. ${ }^{5}$ »Freiheit statt Sozialismus « oder »Freie Fahrt für freie Bürger « lauten die entsprechenden Verballhornungen des Freiheitsverständnisses im Jargon des politischen Alltags. 
Zugleich verrät der abendländische Freiheitsbegriff einen traditionellen Androzentrismus, einen Männlichkeitswahn, weil er ein bestimmtes Bild von einem männlichen Menschen, von Autonomie und Rechtsmacht absolut setzt. So hat in die männliche Menschenrechtserklärung von 1789 bezeichnenderweise eine Formulierung Eingang gefunden, die sich auf die für den Bürger, den Bourgeois, wesentlichste Freiheit bezieht, mit seinem Eigentum nach Belieben zu verfahren. Der Inbegriff bürgerlichen Rechts, das Eigentum an einer Sache, wird auch noch im Bürgerlichen Gesetzbuch als grundsätzlich »unbeschränktes, individuelles Herrschaftsrecht" definiert. Danach kann der Eigentümer einer Sache, »soweit nicht das Gesetz oder Rechte Dritter entgegenstehen, mit der Sache nach Belieben verfahren und andere von jeder Einwirkung ausschließen.« ( $\$ 903$ BGB)

Bei Kant, der in seiner Rechtslehre diesem Rechtsbegriff den wohl präzisesten Ausdruck verliehen hat, heißt es:

Freiheit ist Unabhängigkeit von eines anderen nötigender Willkür ... sofern sie mit jedes anderen Freiheit nach einem allgemeinen Gesetz zusammen bestehen kann ... (sie) ist dieses einzige, ursprüngliche, jedem Menschen kraft seiner Menschheit zustehende Recht.

Wer aber als Mensch gelten kann, wird sofort wieder eingeschränkt, wenn Beispiele für das Verfügungsrecht dieses Rechtssubjektes genannt werden. Neben dem Recht des Besitzes an »körperlichen Sachen« erscheinen »als zur Habe des Subjekts gehörend, sein Weib, Kind und Knecht«. Bis heute entlarvend und im Verständnis der Rechtswissenschaft bestimmend sind seine Definitionen von Rechtssubjektivität, sind Begrifflichkeiten wie »Herrschaft«, »Macht«, »Willkür«, »Gewalt«. Der »freie Wille« ist gleichbedeutend mit »Willensmacht«, ist "machthabender Wille«, versteht sich als »Anmaßung« und ist immer "gegen andere Personen « zu verteidigen. ${ }^{6}$

Ein Vergleich dieser Freiheitsdefinition mit der Frauenrechtserklärung der Olympe de Gouges aus dem Jahr 1791 zeigt, daß die Verfasserin Freiheit explizit mit Gerechtigkeit verbindet. Auffällig ist auch, daß es ihr nicht um die Beanspruchung oder Erweiterung des Freiheitsraumes für Frauen geht, also darum stun und lassen zu können, was einem anderen nicht schadet, « vielmehr fordert sie zurückzuerhalten, „was ihnen (den Frauen) zusteht aufgrund natürlichen Rechts«. ${ }^{7}$

Diese unterschiedlichen Definitionen regen an, zu fragen, warum eigentlich Freiheit in aller Rechtstheorie bisher offenbar nur vorstellbar ist als ein Recht oder ein Raum, der kleiner wird, wenn er mit anderen geteilt, wenn alle anderen auch frei und gleich sind. Warum kommt die/der andere nur als Schranke, Grenze der eigenen Freiheit, nur als ihr Gegner in den Sinn, anstatt die Freiheit der anderen als Erweiterung auch der eigenen Handlungsmöglichkeiten, als mögliche Bereicherung zu denken. "Macht«, selbst »Willensmacht « müßte die Macht einer/eines anderen nicht ausschließen. Vorstellbar ist eine Mächtigkeit, die durch die gleichberechtigte Teilhabe Vieler größer und stärker wird. Solche Überlegungen treffen sich mit der feministischen Moralkritik, die, angeregt durch Carol Gilligan, einer »Ethik der Verantwortung« anstelle einer abstrakten »Moral der 
Rechte « einen mindestens gleichen Rang einräumt und hiermit in der feministischen Theorie und Politik eine breite Debatte über die »zwei Moralen« ausgelöst hat. $^{8}$

In der Präambel zum Verfassungsentwurf des »Runden Tisches « findet sich eine Formulierung, die diesem Freiheitsverständnis entspricht, wenn es heiBt: »daß die Möglichkeit zu selbstbestimmtem verantwortlichen Handeln höchste Freiheit ist. ${ }^{9}$

\section{Gleichheit}

Gleichheit oder Gleichberechtigung ist die zentrale Grundrechtsnorm, die den Kampf um Frauenemanzipation von seinen Anfängen an begleitet. ${ }^{10}$ In der Diskussion um Gleichheit oder Differenz, die gegenwärtig auch die bundesrepublikanische Debatte in der Frauenforschung beherrscht, gibt es unterschiedliche Positionen:

Da sind zum einen die Theoretikerinnen der Gleichberechtigung, eines sog. humanistischen Feminismus ${ }^{11}$, die den aufklärerischen Ansatz zur Emanzipation, die Parole von der Freiheit und Gleichheit aller Menschen, gerade auch der Frauen beim Wort nehmen. Allerdings waren die Maßstäbe für Gleichheit historisch bisher einseitig von männlichen Normen und Maßen bestimmt, und damit ist das Gleichheitsmodell unter der Voraussetzung der Angleichung, der Anpassung an die männliche Normalbiographie oder Lebensweise für Frauen heute verdächtig, unpraktikabel, ja unzumutbar geworden.

Deshalb beharrt die aus der Frauenbewegung gespeiste feministische Rechtsoder Moraltheorie neuerdings sehr viel selbstbewuBter auf der Differenz, der Differenz der Geschlechter als der alle Lebensbereiche, Politik, soziale Teilhabe, Kultur, Sprache, Identität und Wissenschaft prägenden Struktur dieser Gesellschaft. Zur Herausbildung einer autonomen weiblichen Subjektivität, zur Erlangung eines eigenen Raums für eine andere politische Praxis, die nicht unbedingt Separatismus bedeutet, geht es in dieser Perspektive darum, Frauen nicht nur als Unterlegene, Unterdrückte zu denken, vielmehr weibliche Wertmaßstäbe und Orientierungen anzuerkennen und zur Geltung zu bringen.

Allerdings ist die "Aufwertung " von Weiblichkeit als Bezugspunkt einer speziell »weiblichen Freiheit ${ }^{12}$ ambivalent und problematisch, insofern sie der »Verlockung ererbter Weiblichkeit ${ }^{13}$ nicht entgeht, bzw. einer Wesensbestimmung von Weiblichkeit folgt ${ }^{14}$ die doch gerade der historische und gegenwärtige AnlaB der Ungleichbehandlung und Unterdrückung der Frauen war und ist. Am pointiertesten wird diese Position von der französischen Philosophin Luce Irigaray formuliert, wenn sie in ihrem Katalog notwendig "geschlechtsdifferenzierter Rechte « für Frauen z.B. die Unantastbarkeit des weiblichen Körpers und damit das Recht auf »Jungfräulichkeit« oder das Recht auf Mutterschaft als Bestandteil der weiblichen Identität fordert. ${ }^{15}$

In der auf dem Frankfurter Kongre $B$ »Menschenrechte haben (k)ein $\mathrm{Ge}$ schlecht» lebhaft geführten Diskussion über »Gleichheit und/oder Differenz« 
konnten sich die Vertreterinnen der unterschiedlichen Positionen darauf verständigen, daB die Bezugnahme auf Gleichheit oder Differenz keine Alternative und somit eine falsche Wahl wäre. Denn Gleichberechtigung macht nur Sinn unter der Voraussetzung der Verschiedenheit der Menschen, also auch der Verschiedenheit zwischen Männern und Frauen. Andererseits gehen auch die Vertreterinnen einer Differenztheorie implizit bzw. selbstverständlich davon aus, daB die Differenz auch für Frauen nur unter der Voraussetzung gleicher Berechtigungen gelebt werden kann.

Dies bedeutet: Das Gleichheitspostulat geht davon aus, daB Männer und Frauen verschieden sind, daß sie auch infolge der Gleichbehandlung nicht identisch werden, vielmehr ihre Verschiedenheit bewahren können. Die Rede von der "Gleichmacherei«, die immer wiederholte Betonung einer eigentlich nur gemeinten »Gleichwertigkeit«, geht also an der Sache vorbei. Und die Notwendigkeit eines anderen Maßstabes, eines »tertium comparationis« besagt, daB die Gleichberechtigung sich nicht nur nach dem Manne richten, keineswegs nur »Angleichung an die Mannesstellung « bedeuten kann. ${ }^{16}$ Sie muB sich vielmehr an einem übergeordneten Maßstab, einem für alle Menschen (Männer und Frauen) möglichen $\mathrm{MaB}$ von Freiheit orientieren. Sie darf nicht die Arbeits- und Lebensweise von Männern, die sog. männliche Normalbiographie zur Voraussetzung rechtlicher Ansprüche und sozialer Sicherheiten machen. Erst recht aber meint die Forderung nach Rechtsgleichheit nicht Nachahmung all der Fehler, des zerstörerischen Wahnsinns und der Überheblichkeiten, die die Welt in den Abgrund zu reißen drohen.

Trotz des bisher oft mißverstandenen und nicht verwirklichten Gleichheitssatzes, gerade in anbetracht notwendiger Differenzierung und Betonung der Unterschiede möchte ich auf der Forderung nach Gleichberechtigung beharren, weil sie ein politisches Instrument zum Abbau von Privilegien und Gewaltverhältnissen ist. Unter allen Menschenrechten galt und gilt »Gleichheit« immer noch als radikalste und den Nutznießern des Status Quo gefährlichste Forderung. Denn das Postulat gleicher Rechte und Lebenschancen greift bestehende Herrschaftsverhältnisse, Ungerechtigkeiten und soziale Ungleichheit an und verlangt ein immer wieder neues Nachdenken über die Maßstäbe für Recht und Gerechtigkeit.

\section{Die Würde der Frauen}

»Die Würde der Frauen ist antastbar«. Zu erinnern ist an das Plakat aus der neuen Frauenbewegung, das 1975, nach der Aufhebung der Fristenlösung für den Paragraphen 218, zu sehen war: Umgeben von einer Herrenrunde in Richterrobe wurde da ein Frauenakt zur Schau gestellt. Heute ist bei der Auseinandersetzung um den $\S 218$ von der Würde der Frauen längst nicht mehr die Rede, hat eine deutliche und, wie ich meine, bedenkliche Verschiebung zur Rede über den »Schutz ungeborenen Lebens« stattgefunden.

Ich komme hiermit auf ein keineswegs nur »konkretistisches « Problem, ein Spezialproblem der Frauen oder gar »eine Nebensächlichkeit«, die in der Verfassung nichts zu suchen hat. Vielmehr ist die konfliktreiche Frage, ob eine Frau 
selbst und ohne Einmischung anderer über ihre Schwangerschaft entscheiden kann, nicht von ungefähr der Drehpunkt feministischen Aufbruchs zum Widerstand. Denn die Kontrolle der Sexualität und der Gebärfähigkeit der Frau, ihrer Fähigkeit, Menschen großzuziehen und damit die Kontrolle der Reproduktion der Gattung Mensch, waren und sind offenbar bis heute Anlaß und Hebel patriarchalischer Bevormundung und Unterdrückung der Frauen. Früher regelte die »Herrschaft des Mannes im Hause «, das bürgerliche Familienrecht die Kontrolle weiblicher Produktivkraft als »eheliche Pflicht « und legitimierte damit die geschlechtsspezifische Arbeitsteilung. Seit der zumindest formalen Gleichberechtigung auch im Privatrecht, insbesondere im Familienrecht, wird der Eingriff in das Selbstbestimmungsrecht der Frauen über diesen Strafrechtsparagraphen als quasi einer der letzten Bastionen des Patriarchats verteidigt - eine symbolische Politik und, wie wir spätestens seit den Abtreibungsprozessen in Memmingen wieder wissen, trotz Pille und anderer Verhütungsmittel immer noch alltägliches Elend von Frauen.

Aber es geht in diesem traurigen Kapitel jahrhundertelanger Geschlechterpolitik um nichts Geringeres als die Würde der Frauen.

"Würde", um noch einmal eine Definition von Kant zu benutzen, besteht darin, "... in Ansehung des Anspruchs selbst Zweck zu sein, von jedem anderen auch als ein solcher geschätzt und von keinem bloß als Mittel zu anderen Zwecken gebraucht zu werden ... (darin) steckt der Grund der so unbeschränkten Gleichheit der Menschen. ${ }^{17}$

Im Eherecht, in dem bei den bürgerlichen Rechtstheoretikern bekanntlich solche »allgemein « menschlichen und »vernünftigen « Definitionen ihre inkonsequenten Ausnahmen erleiden, wird dann anknüpfend an die verschiedenen Rollen von Mann und Frau im Sexualakt, die Verschiedenheit der Geschlechter auch als rechtliche Ungleichheit begründet. Die Argumentation - am krassesten bei J.G. Fichte, dessen Eherechtslehre (deshalb?) die gesamte Familienrechtstheorie und ihr Verständnis der Ehe als überpersönliche Institution bis heute beeinflußt und geprägt hat - lautet: Weil die Frau »der Natureinrichtung « der Ehe nach »um eine Stufe tiefer steht« als der Mann, kann sie erst dann wieder auf die gleiche Stufe mit ihm gelangen, »indem sie sich zum Mittel der Befriedigung des Mannes macht ...« und - hierin liegt die ganze Dialektik des spezifisch bürgerlichen $\mathrm{Pa}$ triarchalismus, der Liebe nur als einseitige Unterwerfung der Frau denken kann "sie (die Frau) erhält ihre ganze Würde nur dadurch wieder, daß sie es aus Liebe zu diesem Einen getan habe ${ }^{18}{ }^{18}$

Nicht erst in der neuen Frauenbewegung hat sich die Empörung der Frauen an dieser systematischen Kontrolle weiblicher Sexualität entzündet. Helene Stöcker hat z.B. um die Jahrhundertwende ihre "neue Ethik « mit dem Ziel gerade auch sexueller Selbstbestimmung der Frau begründet und antwortete auf Fichte: „Die Frau erlangt ihre Würde in der Hingabe an den Mann nicht dadurch wieder, daB sie es sfür den Einen` tut (wie Fichte meinte), auch nicht dadurch, daß sie Mutter wird « - sondern »durch das Glück gegenseitiger Anerkennung «. ${ }^{19}$

Dies ist aber genau die Denkweise derer, die zum $\gg$ Schutz des werdenden Le- 
bens « wiederum die Frau nur zum Mittel, GefäB, in dem das »andere« Leben wächst, degradieren wollen. In dieser Argumentation wird die körperliche Verbundenheit von Fötus und Schwangerer aufgelöst, der Nasciturus wird mit Hilfe der wissenschaftlich-technischen Entwicklung in der Reproduktionstechnologie gedanklich und faktisch abgetrennt, zu einer eigenen Rechtsposition verselbständigt, seine Rechte als »Rechte anderer« nach dem Prinzip der »Güterabwägung" gegen das Leben, die Existenz der Mutter als Grenze ihrer Entfaltungsfreiheit ausgespielt. ${ }^{20}$ Im Grundgesetzkommentar bei Maunz-Dürig wird die Entpersönlichung, d.h. Entwürdigung bei dieser Güterabwägung so weit getrieben, daB das Lebensrecht der Mutter noch der Rechtfertigung durch ihre weiteren Familienpflichten bedarf. ${ }^{21}$

Es ist das Prinzip der Trennung des Lebendigen, das zusammengehört, des "Teile und Herrsche«, der Kontrolle und Bevormundung, das in diesem Konflikt politisch den Ausschlag gibt. Dabei ist der Vorwand, das Recht der Ungeborenen Kinder gegen die Frauen zu schützen, absurd in einer Gesellschaft, in der die Frauen die Lasten und die Fürsorge der Kindererziehung grundsätzlich allein übernehmen. Bemerkenswert ist, daB z.B. in dem Minderheitsvotum des Bundesverfassungsgerichts zur Fristenlösung die einzige »unterzeichnende Richterin«, Frau Rupp-von-Brünneck, ausdrücklich betont, daB »für das natürliche Empfinden der Frau ... erst in einem längeren Entwicklungsprozeß ein vom mütterlichen Organismus trennbares selbständig existentes Leben entsteht $\ll .{ }^{22}$ Doch anders als in den frühen feministischen Protesten gegen die Entwürdigung der Frauen in dieser ihrer ureigenen Sache, die am Beginn der 1970er Jahre unter dem Slogan "Mein Bauch gehört mir! « auf das Problem aufmerksam machten, ist es heute angemessener und notwendig, den Aspekt der Würde der Frau zu bedenken und zu betonen - die Würde der Frau, die ihre eigene Verwundbarkeit und Antastbarkeit hat -, anstatt mit dem Recht auf »freie Entfaltung der Persönlichkeit« zu argumentieren, das in jedem Fall mißverständlich und wie Freiheit überhaupt auch in anderen Hinsichten problematisch ist.

So endet mein rechtsphilosophischer Exkurs über die Menschenrechte der Frau mit einem sehr aktuellen rechtspolitischen Appell: Sollte nicht allein schon deshalb, weil Männer niemals in ihrem Leben die Erfahrung der Schwangerschaft und der mit ihr verbundenen Notlagen machen, die Entscheidung dem so auffallig anderen RechtsbewuBtsein der Frauen überlassen bleiben und auch der Gesetzgeber endlich das Recht der Selbstbestimmung und Würde der Frau respektieren? 
Im Sinne der Initiative >Frauen für eine neue Verfassung، schließe ich mit dem Aufruf:

In Verbundenheit mit den BürgerInnenbewegungen in der DDR, denen wir die Wende und die Veränderung unserer politischen Verhältnisse verdanken, anknüpfend und solidarisch mit den vielen anderen Initiativen, die für eine grundlegende Diskussion unserer Gesellschaftsverfassung oder einzelne zentrale Forderungen eintreten;

z.B. die Initiative "Frauen in bester Verfassung «, sowie der DGB-Frauen und DDR-Politikerinnen, die öffentlich ein in der Verfassung verankertes Recht auf Arbeit und Kinderbetreuung fordern,

dem Kuratorium für einen demokratisch verfaßten Bund Deutscher Länder und vielen anderen Einzelinitiativen, wie etwa die der »Internationalen Ärzte zur Verhütung eines Atomkrieges«, bundesdeutsche Sektion, die das Verbot des Krieges und der Waffenproduktion, sowie den Schutz der Umwelt in einer neuen Verfassung verankern wollen

fordern wir eine Verfassungsgebende Versammlung, in der endlich und zum erstenmal in der Geschichte Frauen gleichermaßen vertreten sind.

Wesentliche Rechtserrungenschaften in unserer Geschichte sind durch gemeinsamen Protest, durch eine alle Parteien und Vorbehalte überschreitende Mobilisierung zustandegekommen - zu erinnern ist an die gemeinsame Protestversammlung aller Frauenorganisationen der alten Frauenbewegung zur Erlangung des Stimmrechts im Jahr 1918, an die Waschkörbe voller Protestbriefe an den Parlamentarischen Rat 1949, nachdem Elisabeth Selbert zunächst vergeblich versucht hatte, den Gleichberechtigungsartikel in der heutigen Fassung durchzusetzen.

Unsere Diskussion soll ein Anfang sein, gemeinsam mit den Frauen aus der DDR, die seit 1949 eine andere Gleichberechtigungsgeschichte und andere Erfahrungen haben, eine neue Verfassung zu erstreiten.

\section{Anmerkungen}

1 Der Redetext von der Paulskirchenversammlung »Frauen für eine neue Verfassung « vorm 29.9.1990 bezieht sich hier auf den 1989 in Frankfurt durchgefuihrten KongreB »Menschenrechte haben (k)ein Geschlecht«, vgl. hierzu den Dokumentationsband Differenz und Gleichheir, hrsg. v. Ute Gerhard u.a., Frankfurt a.M. 1990, sowie eine Fülle ähnlicher Titel.

2 Ise Frappan: «Wir Frauen haben kein Vaterland*, Berlin 1899

3 taz vom 25.9.90

4 Eine überarbeitete Fassung des folgenden Textes ist inzwischen erschienen in: Leviathan (1991), Nr. 2, S. 4

5 Maunz-Dürig-Herzog u.a., Grundgesetz-Kommentar, München 1990, zu Art. 2 GG, RD.Nr. 25

6 Alle Zitate Immanuel Kant: Metaphysik der Sitten. Erster Teil, Sämtliche Werke, Leipzig 1922, Bd. 5, S. 344 u. $354 \mathrm{ff}$.

7 Vgl. die Gegenüberstellung der männlichen und weiblichen Version in: Ute Gerhard, Gleichheit ohne Angleichung, München 1990, S. 263 f. Anhang.

8 Carol Gilligan: Die andere Stimme. Lebenskonflikte und Moral der Frau. München 1984, S. 171 und 180 . 
9 In freier Selbstbestimmung, hrsg. vom Kuratorium für einen demokratisch verfaBten Bund Deutscher Länder, in Zusammenarbeit mit der Heinrich-Böll-Stiftung, Köln 1990, S. 128.

$10 \mathrm{Vgl}$. hierzu ausfuihrlicher Ute Gerhard: Gleichheit ohne Angleichung, a.a.O., S. $13 \mathrm{ff}$.

11 Vgl. hierzu Elisabeth List: Denkverhälmisse. Feminismus als Kritik. In: Dies.; Herlinde Studer. Denkverhälmisse, Frankfurr/M. 1989, S. 13 f.

12 Liberia delle donne di Milano: Wie weibliche Freiheit entsteht. Eine neue politische Praxis, Berlin 1988.

13 Rossana Rossanda: Differenz und Gleichheit. Eröffnungsvortrag. In: Differenz und Gleichheit, a.a. O., S. 22. Vgl. auch Comelia Klinger: Welche Gleichheit und welche Differenz. In: ebd., S. 112.

14 Siehe Adriana Cavarero: Die Perspektiven der Geschlechtsdifferenz. In: Differenz und Gleichheit, a.a.O., S. $96 \mathrm{ff}$.

15 Luce Irigaray: Über die Notwendigkeit geschlechtsdifferenzierter Rechte. In: Differenz und Gleichheit, a.a.O., S. 341.

16 So aber Maunz-Dürig-Herzog u.a.: Grundgesetz-Kommentar, Art. 3 Abs. 2 GG, Rd.Nr. 3.

17 Immanuel Kant: Mutmaßlicher Anfang der Menschengeschichte, Sämtl. Werke, Leipzig 1922, Bd. 1, S. 277.

18 Johann G. Fichte: Grundlage des Naturrechts nach den Prinzipien der Wissenschafislehre(1796), Hamburg 1960, S. 300-306.

19 Helene Stöcker: Die Liebe und die Frauen, München 1906, S. 159; vgl. hierzu auch Heide Schlüpmann: Radikalisierung der Philosophie. Die Nietzsche-Rezeption und die sexualpolitische Publizistik Helene Stöckers. In: Feministische Studien (1989), H. 2, S. 135-144.

$20 \mathrm{Vgl}$. Wolfgang von den Daele: Der Fötus als Subjekt und die Autonomie der Frau. In: Uta Gerhardt/Yvonne Schütze, Hg., Frauensituation, Frankfurt/M. 1988, S. 189 ff.

21 Maunz-Dürig-Herzog, Grundgesetz-Kommentar, zu Art. 2 Abs. 2, Rd.Nr. 23.

22 BVerfGe v. 25.2.1975, Bd. 39, S. 80. 
